\title{
Focus on the surgical approach to transcatheter aortic valve implantation: Complications, outcome, and preoperative risk adjustment
}

\author{
Maximilian Scherner, MD, ${ }^{a}$ Navid Madershahian, MD, ${ }^{a}$ Svenja Ney, MD, ${ }^{a}$ Kathrin Kuhr, MSc, ${ }^{b}$ \\ Stephan Rosenkranz, MD, ${ }^{c}$ Tanja K. Rudolph, MD, ${ }^{\mathrm{c}}$ Elmar Kuhn, MD, ${ }^{\mathrm{a}}$ Ingo Slottosch, MD, ${ }^{\mathrm{a}}$ \\ Antje Deppe, MD, ${ }^{\mathrm{a}}$ Yeong-Hoon Choi, MD, ${ }^{\mathrm{a}}$ Stephan Baldus, MD, ${ }^{\mathrm{c}}$ and Thorsten Wahlers, $\mathrm{MD}^{\mathrm{a}}$
}

\begin{abstract}
Objective: Perioperative complications in patients undergoing transcatheter aortic valve implantation remain a major issue affecting outcome. Because preoperative risk adjustment remains challenging and a valid scoring system is missing, we sought to determine the incidence of peri- and postprocedural complications of transapical (TA) or transaortic (TAO) access to define the influence of specific complications on early safety, 30-day mortality, and 1-year survival. Furthermore, we aimed to develop a risk-stratification model to allow an estimation of the perioperative risk and the 1year survival rate, based on the individual preoperative condition of each patient.
\end{abstract}

Methods: We performed an outcome analysis of 230 consecutive patients who underwent aortic valve implantation via transapical or transaortic access between 2008 and 2012, with regard to Valve Academic Research Consortium II criteria, including univariate and multivariable regression analysis, to develop a riskstratification model.

Results: Thirty-day mortality was $12.7 \%$. Estimated 1-year survival was 0.69 (95\% confidence interval [CI], 0.631-0.757), and 3-year survival was 0.554 (95\% CI, 0.474-0.634). Univariate logistic regression analysis revealed a significant influence on 30-day mortality in case of life-threatening bleeding (16.1-fold), abdominal complications (8.5-fold), and acute kidney injury (3.2-fold). Pacemaker implantation (odds ratio, $1.55 ; 95 \% \mathrm{CI}, 0.42-5.81 ; P=.512$ ) was not a significant predictor. Concerning use of intraprocedural hemodynamic bridging therapy via cardiopulmonary bypass (CPB), Cox regression analysis revealed no significant survival difference after 1 year. A preoperative risk-stratification model for 1-year survival revealed that a logistic European System for Cardiac Operative Risk Evaluation score $>20 \%$, preoperative existing coronary artery disease, and prior myocardial infarction appeared to be significant predictors for diminished survival.

Conclusions: Concerning intraprocedural complications, CPB support for hemodynamic stabilization is a safe treatment option. Therefore, the heart team approach with CPB standby represents a life-saving option. Attention should also be drawn to specialized and individual postoperative care, because nonprocedure-specific complications clearly affect postoperative short- and long-term outcome. In addition, the risk-stratification model might facilitate preoperative decision making. (J Thorac Cardiovasc Surg 2015;150:841-9)

Patients with symptomatic aortic stenosis carry a poor prognosis if managed nonsurgically-in fact, with occurrence of chest pain, syncope, or any evidence of congestive left heart

\footnotetext{
From the ${ }^{\mathrm{a} D e p a r t m e n t}$ of Cardiothoracic Surgery, ${ }^{\mathrm{b}}$ Institute of Medical Statistics, Informatics, and Epidemiology, and ${ }^{c}$ Department of Cardiology, University Hospital of Cologne, Cologne, Germany.

M.S. and N.M. contributed equally to this work.

Received for publication Sept 28, 2014; revisions received May 9, 2015; accepted for publication June 18, 2015
}

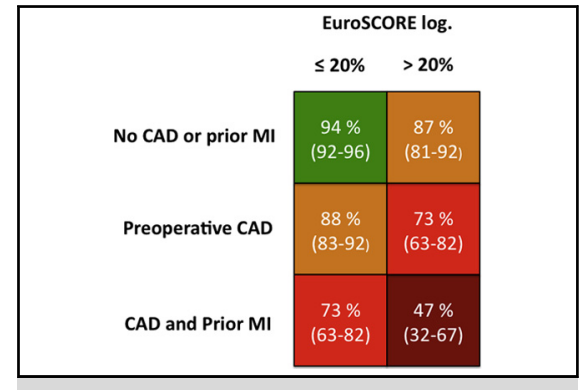

Risk-stratification model to estimate 1-year survival based on preoperative condition.

Central Message

CPB support is a safe treatment option without influence on survival. Nonprocedure-specific complications affect outcome.

\section{Perspective}

The heart team approach with CPB-standby represents a life-saving option in TAVI. Attention should be drawn to specialized postoperative care, because even nonprocedure-specific complications account for short- and longterm outcome. Further efforts to reduce postoperative AR may improve long-term outcome, and the risk-stratification model might facilitate reasonable preoperative decision making.

See Editorial Commentary page 849. failure, mortality rates increase rapidly. Once symptoms occur, severe aortic stenosis carries an average 1-year survival of $60 \%$, whereas 5 -year survival rates average around

\footnotetext{
Address for reprints: Maximilian Scherner, MD, Department of Cardiothoracic Surgery, University Hospital Cologne, Kerpener Strasse 68, 50937 Köln, Germany (E-mail: maximilian.scherner@uk-koeln.de). $0022-5223 / \$ 36.00$

Copyright (C) 2015 by The American Association for Thoracic Surgery http://dx.doi.org/10.1016/j.jtcvs.2015.06.030
} 


$$
\begin{aligned}
& \text { Abbreviations and Acronyms } \\
& \text { AR }=\text { aortic regurgitation } \\
& \text { AVR }=\text { aortic valve replacement } \\
& \text { CAD }=\text { coronary artery disease } \\
& \text { CPB }=\text { cardiopulmonary bypass } \\
& \text { MI }=\text { myocardial infarction } \\
& \text { POD }=\text { postoperative day } \\
& \text { TA }=\text { transapical } \\
& \text { TAO }=\text { transaortic } \\
& \text { TAVI }=\text { transcatheter aortic valve implantation } \\
& \text { TF }=\text { transfemoral } \\
& \text { VARC }=\text { Valve Academic Research Consortium }
\end{aligned}
$$

$32 \% .^{1}$ Therefore aortic valve replacement (AVR) is the only maintainable therapy to improve survival. ${ }^{2}$ Transcatheter aortic valve implantation (TAVI) has been established as an alternative procedure in cardiovascular medicine for the treatment of nonoperable or high-risk patients with severe aortic stenosis. Over the past years, TAVI using transfemoral (TF), transapical (TA), or transaortic (TAO) access has become an established procedure performed worldwide in highly qualified cardiac centers with satisfactory short- and midterm results. ${ }^{3}$ Although experience and techniques have constantly improved over the past years, peri- and postprocedural complications in high-risk TAVI collectives remain a major issue affecting outcome and survival. ${ }^{4}$ Because the TF approach is considered the first option at most sites, surgical TAVI via TA or TAO access is mostly performed in patients with even higher preoperative morbidity. ${ }^{5,6}$ Consequently, patients undergoing surgical TAVI represent a specific population among the patients selected for transcatheter valve therapies, and should therefore be analyzed individually. Moreover, preoperative risk adjustment remains challenging in these patients as long as a valid scoring system is missing. We sought to determine the incidence of periprocedural complications of TA/TAO aortic valve implantation and to define the influence of specific complications on 30-day mortality and 1-year survival. Additionally, we aimed to develop a risk stratification model to allow an estimation of patients' 1-year survival probability based on the preoperative condition.

\section{PATIENTS AND METHODS \\ Data Collection and Preoperative Dispositions}

Two hundred thirty consecutive patients who underwent TA/TAO-TAVI between January 2008 and December 2012 at our institution due to severely increased risk for conventional AVR therapy were included in this study. All data were prospectively collected in our institutional database and retrospectively analyzed. Patient demographic and preoperative characteristics are provided in Table 1. We included all patients of our TAVI program and statistically adjusted for a possible learning curve effect (see Statistical Analysis section). Mean follow-up was 1.5 years (range, 0 days-4.0 years). All patients accepted for TA-TAVI and TAO-TAVI were reviewed by an interdisciplinary team based in our institution (ie, the heart team) who estimated frailty, preoperative cardiac status, and preexisting comorbidities representing the risk profile of each individual patient. TF-TAVI was considered as the first option (TF-first strategy), whereas TA or TAO access was considered in case of contraindications for TF-TAVI. TAO-TAVI has been performed since 2010 in case of expected disadvantages of TATAVI (eg, pleural adhesions, severe chronic obstructive pulmonary disease, or left ventricular aneurysm). Local ethics committee approval was obtained for this study. None of our patients were planned for intraoperative cardiopulmonary bypass (CPB) support. All procedures were performed in a hybrid operating room with CPB as emergency standby.

\section{Prosthetic Valve System and Procedure}

TA and TAO-TAVI were performed using the Edwards Sapien XT prosthesis $(\mathrm{n}=219)$ (Sapien; Edwards Lifesciences, Irvine, Calif), Corevalve $(\mathrm{n}=5)$ (Corevalve; Medtronic, Minneapolis, Minn) or Symetis $(\mathrm{n}=5)(\mathrm{Sy}-$ metis; Accurate TA, Ecublens, Switzerland). Information concerning the prosthetic valve system was not available for 1 patient. Procedure steps were generally performed as previously described, ${ }^{7}$ and were adapted for the individual valve type and system.

\section{End Points}

The objectives of this study were to analyze the incidence of periprocedural complications of TA/TAO-TAVI, to analyze the influence of specific complications on 30-day mortality and 1-year survival, and to develop a riskstratification model to allow an estimation of the calculated 1-year survival probability based on the preoperative condition. Survival was defined as date of operation to death of any cause or to the date of last information. To perform accurate risk factor analysis for diminished 1-year survival, we excluded all events (mortality) that occurred until postoperative day (POD) 30 to exclude a bias based on early complications. Therefore, 2 postoperative periods were analyzed: day of operation to POD 30, and POD 31 to 365.

\section{Statistical Analysis}

Pre-, peri-, and postoperative factors were described using mean values \pm standard deviation, median (25th-75th percentile), or frequencies and percentages. Survival was estimated by the Kaplan-Meier method. The influence of periprocedural risk factors on 30-day mortality was analyzed using univariate logistic regression models (out of 221 patients), the influence on 1-year survival using univariate Cox regression models (out of 193 patients), respectively. Models were fitted for risk factors that occurred in $>10$ patients. To develop a risk-stratification model for 1-year survival based on the preoperative risk profile, a multivariable Cox regression analysis was performed. Clinically relevant preoperative covariates showing a $P$ value $<.05$ in the univariate analysis and remaining in the model after backward, stepwise selection procedure were included in the final model. Variables considered are given in Table 1. Prior myocardial infarction (MI) was defined as a substantiated myocardial event in a patient's medical history, but no acute infarction or occurrence of severe symptoms within the immediate preoperative course (last 90 days before surgery). Because coronary artery disease $(\mathrm{CAD})$ and prior $\mathrm{MI}$ both remained in the model we created a new covariate with 3 categories (ie, neither CAD nor MI, isolated $\mathrm{CAD}$, and $\mathrm{MI}$ ) to account for the fact that in general patients with prior MI also experienced CAD. The fitted model was used to estimate the 1-year survival rate, with respect to the identified risk factors. For 30-day mortality, a multivariable analysis was not assessable due to the low number of deaths that occurred. For logistic regression models, odds ratios (ORs), corresponding 95\% confidence intervals (CIs), and $P$ values (Wald test) were given. For Cox regression models, hazard ratios (HRs), corresponding $95 \% \mathrm{CIs}$, and $P$ values (Wald test) were reported. Proportional hazards assumption was checked graphically. All fitted regression models included the year of operation (2008 vs 2009-2012) as covariate to account for possible learning curve effect. Sensitivity analyses comparing patients treated during $2008(\mathrm{n}=35)$ with patients treated 
TABLE 1. Baseline characteristics of patients undergoing transcatheter aortic valve implantation $(\mathrm{N}=\mathbf{2 3 0})$

\begin{tabular}{lc}
\multicolumn{1}{c}{ Variable } & Result \\
\hline Clinical characteristic & \\
Male gender & $96(41.74)$ \\
Age (y) & $81.62 \pm 6.19$ \\
Body mass index & $26.43 \pm 4.50$ \\
European System for Cardiac Operative Risk & $18.92(11.60-30.89)$ \\
$\quad$ Evaluation score (\%) & \\
$\quad>20 \%$ & $105(47.73)$ \\
Society of Thoracic Surgeons predicted risk & $5.48(3.77-8.40)$ \\
$\quad$ of mortality (\%) & \\
New York Heart Association functional & $195(85.53)$ \\
$\quad$ class III or IV & \\
Coronary artery disease & $142(61.74)$ \\
Chronic obstructive pulmonary disease & $66(28.70)$ \\
Peripheral vascular disease & $60(26.09)$ \\
Atrial fibrillation & $79(34.35)$ \\
Diabetes & $79(34.35)$ \\
Arterial hypertension & $208(90.43)$ \\
Pulmonary hypertension & $64(27.83)$ \\
Hyperlipidemia & $109(47.39)$ \\
Prior myocardial infarction* & $40(17.39)$ \\
Cerebrovascular disease & $59(25.65)$ \\
Pacemaker preoperatively & $26(11.30)$ \\
Prior cardiac surgery & $92(40.00)$ \\
Hemoglobin (mg/dL) & $12.50(11.30-13.50)$ \\
$\quad<12$ mg/dL & $87(37.99)$ \\
Echocardiographic data & \\
Preoperative left ventricular ejection & $105(45.65)$ \\
$\quad$ fraction (\%) & $1.07(0.83-1.48)$ \\
Aortic stenosis class III-IV & $10(4.35)$ \\
Aortic regurgitation & $54.04 \pm 14.94$ \\
Mitral stenosis & \\
Mitral regurgitation & $226(98.26)$ \\
Preoperative renal impact & $158(68.7)$ \\
Preoperative serum creatinine (mg/dL) & $10(4.35)$ \\
Preoperative dialysis & $36(15.72)$ \\
\hline
\end{tabular}

Values are presented as $\mathrm{n}(\%)$, mean \pm standard deviation, or median (25th-75th percentile). *Prior myocardial infarction was defined as a substantiated myocardial event in a patient's medical history, but no acute infarction or occurrence of severe symptoms within the immediate preoperative course (last 90 days before surgery). $\dagger$ Defined as creatinine $>1.1 \mathrm{mg} / \mathrm{dL}$

between 2009 and $2012(\mathrm{n}=195)$ yielded similar results for both groups regarding relevant outcome modalities (data not shown). All reported $P$ values are 2 -sided. Because the analyses were regarded as explorative we did not adjust for multiple testing. Statistical analyses were performed using IBM-SPPS Statistics version 22 (Armonk, NY), and R version 3.0.2 (R Foundation for Statistical Computing, Vienna, Austria).

\section{RESULTS}

\section{Intraprocedural Course}

Procedural success was achieved in $97.9 \%$ of all cases. Nine patients $(3.9 \%)$ received an unplanned valve-in-valve implant due to significant paravalvular leak, whereas $10 \mathrm{pa}-$ tients $(4.4 \%)$ underwent an additional intraoperative balloon dilation. Emergency cardiac surgery (defined as emergency implementation of CPB either as intraoperative bridging or in combination with conversion to conventional AVR) was performed in $10.4 \%$ of patients. Nine patients $(3.9 \%)$ required emergency conversion to conventional AVR. Eight of those underwent conversion intraoperatively, whereas 1 patient decompensated during POD 1 due to secondary valve dislocation. Fifteen patients $(6.5 \%)$ needed intraoperative CPB support due to hemodynamic instability. Twenty-three patients needed cardiopulmonary resuscitation during the procedure due to hemodynamic instability. With respect to fatal intraprocedural complications $(n=3), 1$ patient died due to left ventricular perforation, 1 patient experienced acute left ventricular rupture followed by emergency conversion and consecutive low cardiac output syndrome, and 1 patient died after emergency conversion due to valve dislocation that occurred on the first POD. All 3 patients died despite the implementation of extracorporeal membrane oxygenation therapy. With regard to the immediate prosthetic valve performance, $93.5 \%$ showed none or trace aortic regurgitation (AR), whereas 14 patients $(6.1 \%)$ presented a postoperative AR grade II. In these cases, AR $>$ grade I was accepted due to extensive left ventricular outflow tract or annulus calcification representing a high risk for annular rupture. One patient $(0.4 \%)$ developed AR grade IV due to postprocedural valve dislocation requiring repeat procedure. With regard to the influence of emergency cardiac surgery, unplanned CPB use without conversion was not associated with increased 30-day mortality $(P=.718)$. A detailed overview as well as further procedural data are given in Tables 2-5.

\section{Postprocedural Course (30-Day Outcome)}

Total 30-day-mortality was $12.7 \%(\mathrm{n}=28)$. Acute kidney injury was the most frequent postoperative complication $(45.3 \%)$, followed by respiratory insufficiency with need for reintubation (14.9\%), life-threatening bleeding complications $(14.4 \%)$, postoperative atrioventricular block requiring pacemaker implantation $(7.4 \%)$, stroke $(6.1 \%$; major stroke, $2.2 \%$ ), and pneumonia (5.2\%). Abdominal complications (eg, ileus, subileus, or any form of acute abdomen distress) occurred in $5.2 \%$ and led to laparotomy in 5 patients $(2.2 \%)$. The median postoperative catecholamine support was 13 hours for dobutamine (25th-75th percentiles, 2.0033.00 ) and 8 hours for noradrenaline (25th-75th percentiles, 3.00-22.50). The median intensive care unit stay was 3.00 days (25th-75th percentiles, 2.00-6.00) and the median hospital stay was 11 days (25th-75th percentiles, 8.00-13.00).

Univariate analysis revealed a significant influence on 30day mortality in case of life-threatening bleeding complications (16.1-fold; OR, 16.1; 95\% CI, 6.34-41.07; $P<.001)$. In line with this, rethoracotomy (3.5-fold; OR, $3.5 ; 95 \%$ CI, 1.11-11.22; $P=.033)$ and red blood cell transfusion $\geq 4$ units (11.7-fold; OR, $11.7 ; 95 \%$ CI, 4.6729.38; $P<.001)$ were associated with a significantly increased 30-day mortality risk. Moreover abdominal 
TABLE 2. Peri- and postprocedural data according to Valve Academic Research Consortium end points $(\mathbf{N}=\mathbf{2 3 0})$

\begin{tabular}{lc}
\hline \multicolumn{1}{c}{ Variable } & Result \\
\hline Periprocedure & \\
Postdilation & $10(4.35)$ \\
Valve-in-valve implantation (unplanned) & $9(3.91)$ \\
Coronary obstruction & $0(0)$ \\
Emergency surgery* & $24(10.43)$ \\
Cardiopulmonary bypass use (unplanned) & $15(6.52)$ \\
Conversion to conventional aortic valve repair & $9(3.91)$ \\
Aortic regurgitation $>1$ & $15(6.52)$ \\
Grade II & $14(6.09)$ \\
Grade III & $0(-)$ \\
Grade IV & $1(0.43)$ \\
Intra-aortic balloon pump implantation & $6(2.61)$ \\
Extracorporeal membrane oxygenation implantation & $3(1.30)$ \\
Postprocedure & \\
Combined 30-d early safety end point & $72(32.14)$ \\
Mortality (30 d) & $28(12.67)$ \\
Neurologic event & $14(6.09)$ \\
Minor stroke & $9(3.91)$ \\
Major stroke & $5(2.17)$ \\
Life-threatening bleeding & $33(14.35)$ \\
Acute kidney injury & $96(45.28)$ \\
Stage I & $61(28.77)$ \\
Stage II & $9(4.25)$ \\
Stage III & $26(12.26)$ \\
Vascular complication & $4(1.74)$ \\
Permanent pacemaker implantation & $17(7.39)$ \\
New third-degree atrioventricular block & $17(7.39)$ \\
Myocardial infarction & $1(0.43)$ \\
Device success & $174(75.65)$ \\
\hline Values are presented as n (\%). *Emergency surgery was defined as unplanned need \\
for cardiopulmonary bypass due to hemodynamic instability or conversion from \\
transcatheter valve replacement to conventional aortic valve replacement. \\
\end{tabular}

complications (8.5-fold; OR, 8.5; 95\% CI, 2.53-28.74; $P=$ .001 ), pneumonia (6-fold; OR, 6.0; 95\% CI, 1.76-20.72; $P$ $=.004)$, reintubation $(5.9$-fold; OR, 5.9; 95\% CI, 2.45$14.02 ; P<.001$ ), and acute kidney injury (stages I-III, 3.2fold; OR, 3.2; 95\% CI, 1.17-8.66; $P=.023$ ) appeared to be significant predictors for an increased 30-day mortality risk. Additionally (although statistically not valuable), patients who underwent laparaotomy showed poor survival: laparotomy was performed in 4 patients due to abdominal abnormalities; 3 patients died within the early postoperative course. Neither stroke ( $\mathrm{n}=14$; no deaths), nor pacemaker implantation (OR, 1.55; 95\% CI, 0.42-5.81; $P=.512$ ) was significantly associated with a higher 30-day mortality.

\section{Overall Survival and Risk Factors for 1-Year Mortality}

Estimated 30-day survival was 0.871 (95\% CI, 0.8260.916), 1-year survival was 0.69 (95\% CI, 0.631-0.757), and 3-year survival was 0.554 (95\% CI, 0.474-0.634) (Figure 1). With regard to the coherency between emergency CPB use and 1-year survival, univariate analysis revealed no
TABLE 3. Peri- and postprocedural data additional to Valve Academic Research Consortium II criteria ( $\mathbf{N}=\mathbf{2 3 0})$

\begin{tabular}{lc}
\hline \multicolumn{1}{c}{ Variable } & Result \\
\hline Periprocedure & \\
Surgical access & \\
$\quad$ Transapical & $223(96.96)$ \\
$\quad$ Transaortal & $7(3.04)$ \\
Duration of surgery (min) & $88.0(74.0-111.0)$ \\
Amount of contrast agent (mL) & $85.0(59.0-116.0)$ \\
Cardiopulmonary resuscitation intraoperatively & $23(10.0)$ \\
Postprocedure & \\
Postoperative left ventricular ejection fraction $(\%)$ & $55.04 \pm 12.20$ \\
Rethoracotomy & $17(7.42)$ \\
Red blood cell transfusion $\geq 4$ units & $51(22.37)$ \\
Cardiopulmonary resuscitation postoperatively & $15(6.52)$ \\
Red blood cell transfusion & $150(65.79)$ \\
Dialysis postoperatively & $29(12.61)$ \\
Transition syndrome & $48(20.87)$ \\
$\quad$ Mild & $25(10.87)$ \\
$\quad$ Severe & $23(10.00)$ \\
Abdominal complication & $12(5.22)$ \\
Laparotomy & $5(2.17)$ \\
Pulmonary embolism & $1(0.43)$ \\
Reintubation & $34(14.85)$ \\
Tracheotomy & $5(2.17)$ \\
Pneumonia & $12(5.24)$ \\
Antibiosis & $117(50.87)$ \\
Arterenol (h) & $8(3.00-22.25)$ \\
$\geq 24$ h & $55(24.34)$ \\
Dobutrex (h) & $13(2.00-33.00)$ \\
$\geq 24$ h & $69(30.53)$ \\
Intensive care unit stay (d) & $3.0(2.0-6.0)$ \\
In-hospital stay (d) & $11.0(8.0-13.0)$ \\
\hline Values are presented as $n$ (\%) or median (25th-75th percentile). \\
\end{tabular}

influence of unplanned CPB use (OR, 0.462; 95\% CI, 0.113$1.893 ; P=.283$ ) on 1-year mortality (Figure 2, A). In contrast, 8 out of 9 patients who underwent emergency conversion did not survive the first postoperative year.

With regard to postprocedure complications, univariate Cox regression revealed that abdominal complications (HR, $3.83 ; 95 \% \mathrm{CI}, 1.17-12.55 ; P=.027)$ were significantly associated with an increased 1-year mortality risk. Additionally, residual AR $>$ grade I resulted in a 4.1-fold increased 1-year mortality (95\% CI, 1.72-9.93; $P=.002)$. Neither pacemaker implantation, nor life-threatening bleeding or reintubation were associated with significantly increased 1-year mortality (Tables 4 and 5). Patients requiring postoperative catecholamine support (dobutamine or noradrenaline $>24$ hours) showed a significantly diminished 1-year survival, even after exclusion of the acute postoperative period (day 0-30) (Figure 2, $B$ and $C$ ).

\section{Risk Stratification}

To develop a risk-stratification model for 1-year survival based on the preoperative risk profile, multivariable 
TABLE 4. Univariate regression analysis of Valve Academic Research Consortium II criteria end points

\begin{tabular}{|c|c|c|c|c|}
\hline \multirow[b]{2}{*}{ Variable } & \multicolumn{2}{|c|}{ 30-d Mortality $(\mathrm{n}=\mathbf{2 2 1})$} & \multicolumn{2}{|c|}{ 1-y Mortality* $(n=193)$} \\
\hline & OR $(95 \%$ CI $)$ & $P$ value & HR $(95 \%$ CI $)$ & $P$ value \\
\hline \multicolumn{5}{|l|}{ Periprocedure data } \\
\hline \multicolumn{5}{|l|}{ Prosthetic valve-associated end points } \\
\hline New third-degree atrio-ventricular block & $1.55(0.42-5.81)$ & .512 & $1.19(0.36-3.87)$ & .778 \\
\hline New pacemaker implantation & $1.55(0.42-5.81)$ & .512 & $1.19(0.36-3.87)$ & .778 \\
\hline Aortic regurgitation $>1$ & $2.14(0.54-8.51)$ & .282 & $4.13(1.72-9.93)$ & .002 \\
\hline \multicolumn{5}{|l|}{ Postprocedure data } \\
\hline Life-threatening bleeding complication & $16.13(6.34-41.07)$ & $<.001$ & $0.96(0.29-3.17)$ & .948 \\
\hline Acute kidney injury (stage I-III) $\dagger$ & $3.18(1.17-8.66)$ & .023 & $1.39(0.71-2.72)$ & .342 \\
\hline
\end{tabular}

regression analysis was performed and revealed that a logistic European System for Cardiac Operative Risk Evaluation score $>20 \%(\mathrm{HR}, 2.38 ; 95 \% \mathrm{CI}, 1.14-4.98 ; P=.021)$ and higher cardiovascular risk were independent significant predictors for 1-year mortality; patients with isolated CAD (HR, $2.21 ; 95 \% \mathrm{CI}, 0.85-5.71 ; P=.103)$ and those with CAD with subsequent prior MI (HR, 5.27; 95\% CI, 1.98-14.05; $P=.001$ ) had a higher risk of dying within 1 year postTAVI compared with patients with no history of MI or CAD (overall $P=.003$ ). Correspondingly, Kaplan-Meier analysis revealed a significantly diminished 1-year survival (POD 31-365) in patients with CAD and prior MI (Figure 2, $D$ and $E$ ). We calculated the probability of 1year mortality in dependency on the identified risk factors. Figure 3 gives an overview of the calculated survival probability. The survival probability ranges from 94\% (95\% CI, $92 \%-96 \%$ ) in a patient with a logistic System for Cardiac Operative Risk Evaluation score $<20 \%$ without prior MI or isolated CAD down to $47 \%$ (95\% CI, 32\%-62\%) in a patient with a history of prior MI and a logistic System for Cardiac Operative Risk Evaluation score $>20 \%$.

\section{DISCUSSION}

Our analysis focuses on the outcomes and complications after surgical TAVI, especially with regard to TA access. The implementation of the TA approach is still the subject of debate, and center-specific policies range from a preference for TA aortic valve implantation on 1 hand to TF-first strategies on the other hand. We aimed to analyze the results of surgical TAVI with regard to our center-specific policy and the analyzed patient cohort. The analysis describes a highrisk patient population of all comers in a real-world setting after exclusion of the initial learning curve period. All patients underwent interdisciplinary heart team discussion and were evaluated for conventional AVR, the TF-approach (ie, TFfirst-strategy), or alternative approaches. Therefore, the analyzed patient population represents a very specific cohort in terms of comorbidities and clinical characteristics. The observed 30 -day mortality of $12.7 \%$ is significantly higher than outcomes of the Placement of Aortic Transcatheter Valve (PARTNER) trial, ${ }^{8,9}$ but is in line with other realworld experiences. ${ }^{5,10-12}$ Fifty-four percent of our 30-day mortality was directly related to the procedure itself, whereas the other patients died due to postprocedural complications. The combined safety end point (ie, composite of all-cause mortality, stroke, life-threatening bleeding, acute kidney injury stage II or III, coronary obstruction, major vascular complication, and repeat procedure required by valverelated dysfunction) was met by $32.1 \%$ of patients. Because $12.7 \%$ of the events were deaths, the remaining $19.4 \%$ were represented by other complications.

\section{Intraprocedural Complications}

Severe intraprocedural complications (eg, unplanned valve-in-valve, unplanned $\mathrm{CPB}$, or conversion) requiring immediate surgical or interventional management occurred

TABLE 5. Univariate regression analysis of risk factors in addition to Valve Academic Research Consortium II criteria end points

\begin{tabular}{|c|c|c|c|c|}
\hline \multirow[b]{2}{*}{ Risk factor } & \multicolumn{2}{|c|}{ 30-d Mortality $(n=221)$} & \multicolumn{2}{|c|}{ 1-y Mortality* $(n=193)$} \\
\hline & OR $(95 \%$ CI $)$ & $P$ value & HR $(95 \%$ CI $)$ & $P$ value \\
\hline Rethoracotomy & $3.52(1.11-11.22)$ & .033 & $0.84(0.20-3.55)$ & .817 \\
\hline Red blood cell transfusion $\geq 4$ units & $11.71(4.67-29.38)$ & $<.001$ & $0.96(0.39-2.37)$ & .937 \\
\hline Dialysis postoperatively & $13.19(5.03-34.57)$ & $<.001$ & $1.21(0.37-3.98)$ & .760 \\
\hline Cardiopulmonary resuscitation intraoperatively & $4.04(1.47-11.08)$ & .007 & $1.57(0.55-4.44)$ & .399 \\
\hline Reintubation & $5.86(2.45-14.02)$ & $<.001$ & $1.27(0.50-3.27)$ & .616 \\
\hline Pneumonia & $6.04(1.76-20.72)$ & .004 & $-\dagger$ & - \\
\hline Abdominal complication & $8.52(2.53-28.74)$ & .001 & $3.83(1.17-12.55)$ & .027 \\
\hline Arterenol $\geq 24 \mathrm{~h}$ & $5.93(2.53-13.87)$ & $<.001$ & $3.42(1.76-6.66)$ & $<.001$ \\
\hline Dobutrex $\geq 24 \mathrm{~h}$ & $1.66(0.72-3.83)$ & .230 & $3.10(1.60-5.98)$ & .001 \\
\hline
\end{tabular}

$O R$, Odds ratio; $C I$, confidence interval; $H R$, hazard ratio. *Defined as postoperative day 31-365. †Requirements for Cox regression not fulfilled. 


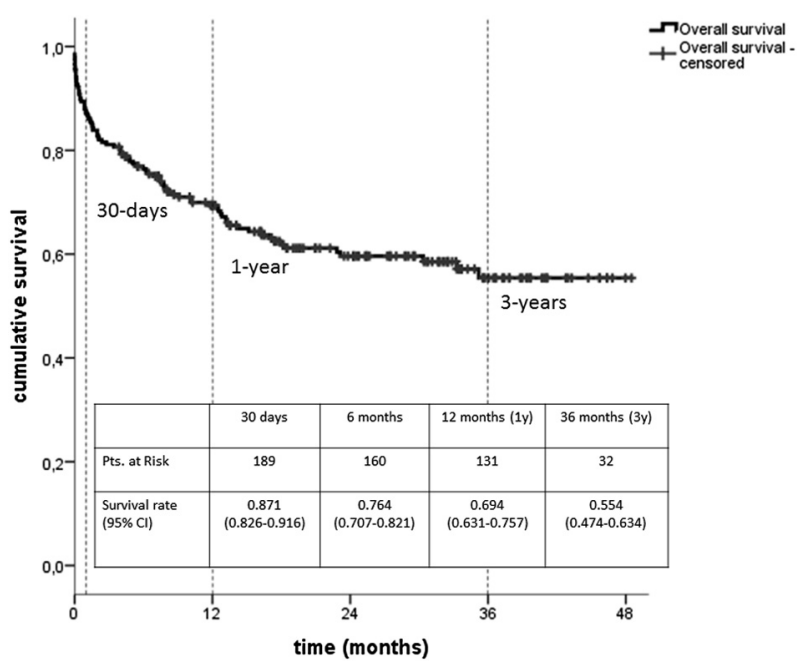

FIGURE 1. Kaplan-Meier test results for overall survival (3 y). Pts, Patients; $C I$, confidence interval.

in $14.3 \%$ of patients. These numbers seem rather high when compared with other series, ${ }^{13}$ but may be explained by the distinct high-risk profile of our patients in addition to a liberal policy towards CPB bridging. Therefore, the majority of intraprocedural complications occurred due to hemodynamic instability requiring $\mathrm{CPB}$ support via femoral cannulation $(6.5 \%)$, followed by unplanned valve-in-valve implantation (3.9\%) and emergency conversion to conventional AVR (3.9\%). Noticeably, CPB use did not appear as a predictor for 30-day or 1-year mortality. In line with this, Kaplan-Meier survival analysis revealed that patients who underwent CPB bridging showed no significantly diminished 1-year survival. In contrast, conversion $(3.9 \%$; reasons included left ventricular outflow tract rupture $[\mathrm{n}=2]$, annulus rupture $[\mathrm{n}=3$ ], and valve dislocation [intraoperatively: $\mathrm{n}=2$; on the first postoperative day: $\mathrm{n}=1$ ]) was associated with a distinct postoperative mortality rate $(66.7 \%)$. These findings are in line with a recently reported meta-analysis of 46 studies $^{14}$ reporting a mortality of $67 \%$ after emergency heart surgery. Although these numbers seem very high, the reasons for emergency conventional surgery reflect procedure-specific complications. In contrast, the outcome of emergency CPB bridging is particularly influenced by the preoperative condition. Therefore, these numbers are specific for a high-risk patient clientele and cannot be applied to patients with different preoperative characteristics.

Unplanned valve-in-valve implantation was performed in $3.9 \%$ and was mainly required because of difficulties concerning an adequate placement of the implanted valve leading to inadequate sealing of the transcatheter heart valve. Because our institutional policy includes a liberal attitude with regard to the implantation of a second valve, these numbers are slightly but not significantly higher when compared with other studies reporting rates between 1.1 and $3.6 \% .^{8,15-19}$ In line with this, $4.4 \%$ of patients underwent a second balloon dilation with repeated rapid pacing. Neither unplanned valve-in-valve implantation nor a second dilation caused relevant complications and therefore can be considered safe treatment options. The influence of postoperative AR on 1-year mortality is still a matter of debate. Our findings are in line with other reports, indicating a more than 4-fold increased risk for 1-year mortality in case of postoperative AR $>$ grade $\mathrm{I} .{ }^{17}$

\section{Postoperative Complications}

We analyzed postoperative complications with regard to the Valve Academic Research Consortium (VARC)-II criteria.

In addition to the criteria defined by VARC, we analyzed the incidence of further postoperative complications not specific to the TAVI procedure itself. Concerning the VARC end points, acute kidney injury was the most frequent complication, which is in line with other studies, $, 20,21$ and was associated with a 3.2-fold increased risk of 30-day mortality after TAVI. Life-threatening bleeding was present in $14.4 \%$ of patients and also represented a predictor for 30-day mortality. Because the definitions for bleeding events according to VARC are very strict, particularly with regard to the surgical approach, and center-specific transfusion policies may influence this parameter, these results have to be interpreted with caution. However, life-threatening bleeding was associated with a 16-fold increased risk for 30-day mortality. Pacemaker implantation was necessary in only $7.4 \%$ of patients. This may be partly explained by the use of the Edwards Sapien XT prosthesis in the vast majority of cases but still represents a low incidence of pacemaker implantation after TA-TAVI compared with other studies. ${ }^{22,23}$

Abdominal complications (eg, ileus, subileus, or any form of acute abdomen distress) were found to be a predictor for 30-day mortality. Moreover, postoperative pneumonia showed a 7-fold increased risk for 30-day mortality. Interestingly, the need for catecholamine support $>24$ hours postoperatively was associated with significantly higher 1-year mortality rates, even with respect to a period under observation starting on POD 31. These findings might appear to be a matter of course with regard to the analyzed patients undergoing TA/TAO-TAVI. However, they underline the importance of exact postoperative care and might be an additional argument to treat these patients in highly specialized and experienced centers. Although these observations only reflect the labile state of these patients, they facilitate postoperative assessment concerning their further postoperative course.

\section{Risk Stratification}

Considering preoperative risk assessment, an elaborate scoring system for TAVI patients is still missing. Moreover, patients undergoing TA/TAO-TAVI (providing that a TFfirst strategy is implemented) harbor a distinctly higher 

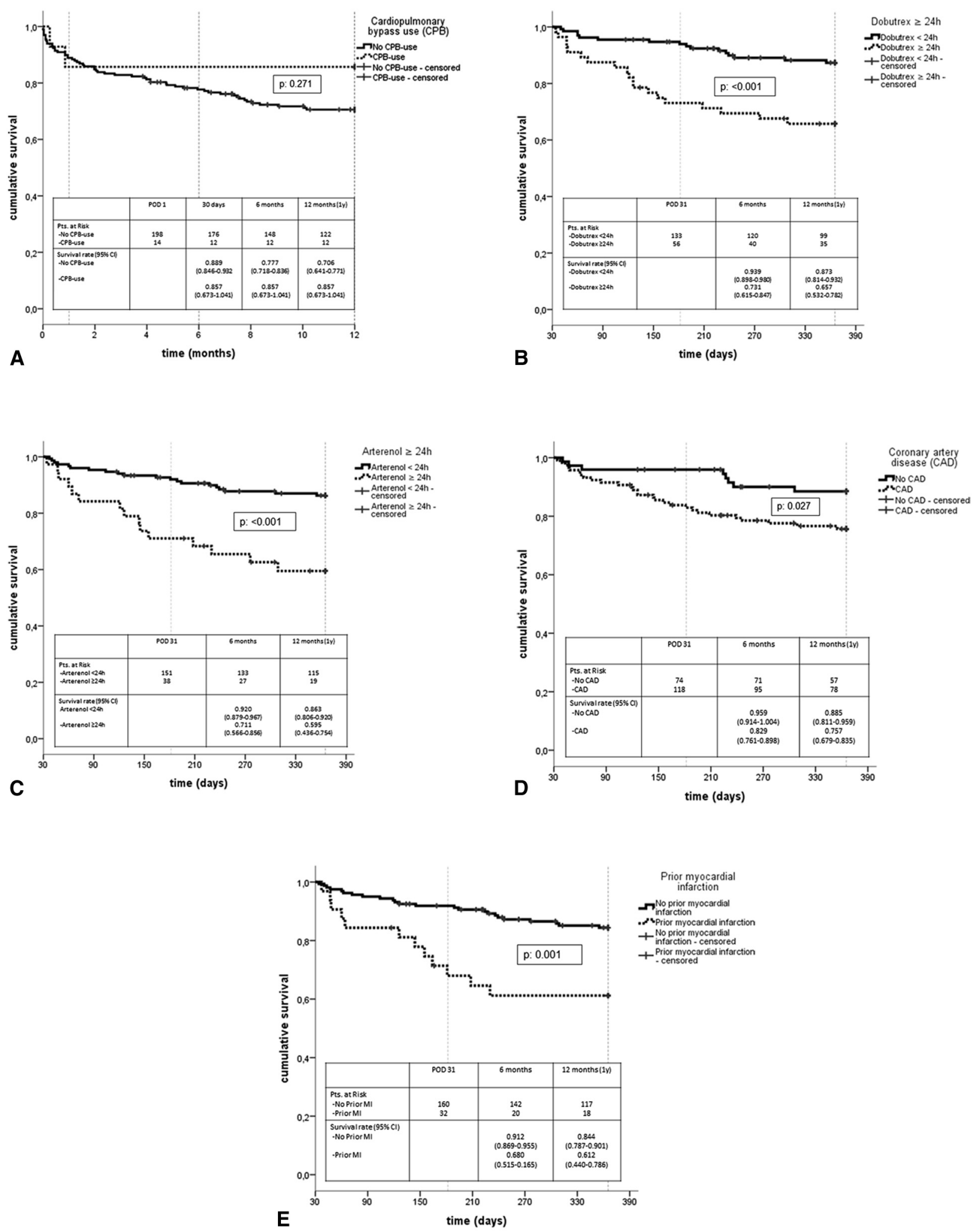

FIGURE 2. A, Kaplan-Meier test results for unplanned cardiopulmonary bypass use (1-y survival). B and C, Kaplan-Meier test results for catecholamine administration (1-y survival). D, Kaplan-Meier test results for coronary artery disease (1-y survival). E, Kaplan-Meier test results for prior myocardial infarction (1-y survival). Pts, Patients; $C I$, confidence interval; $M I$, myocardial infarction; $P O D$, postoperative day. 


\section{EuroSCORE log.}

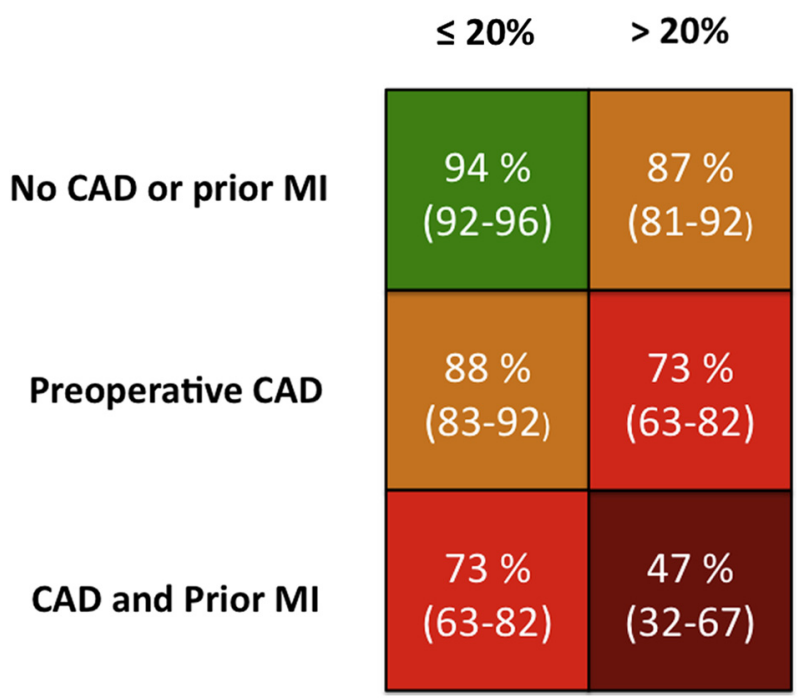

FIGURE 3. Risk-stratification model to estimate 1-year survival. Values are calculated risks as well as $95 \%$ confidence intervals. No risk factors present (green boxes), 1 risk factor present (orange boxes) 2 risk factors present (light red boxes), and 3 risk factors present (dark red boxes). Euro$S C O R E$, European System for Cardiac Operative Risk Evaluation; $C A D$, coronary artery disease; $M I$, myocardial infarction.

preoperative risk profile ${ }^{5,6}$ and might need a different preoperative risk assessment in comparison to patients undergoing the procedure with TF access. To develop a risk stratification model for 1-year survival, we performed multivariable logistic regression analysis to identify statistically independent risk factors. We decided to include established scoring systems in our model (Society of Thoracic Surgeons predicted risk of mortality $\leq />10 \%$ and European System for Cardiac Operative Risk Evaluation score $\leq />20 \%$ ) because these scores are commonly and routinely used for preoperative decision making. With regard to the risk model for the 1-year survival probability, a logistic European System for Cardiac Operative Risk Evaluation score $>20 \%$ and preexisting CAD as well as a prior MI appeared to be significant predictors for diminished survival. The model has to be interpreted as follows (Figure 3): If none of the identified risk factors are present (items in green boxes), the 1-year survival probability is $94 \%$. In case of the presence of 1 risk factor (orange boxes), the probability is around $88 \%$ and decreases to $73 \%$ if a patient presents 2 of the named risk factors (light red boxes). In case of a patient presenting all 3 risk factors the probability decreases and appears to be only $47 \%$ (dark red boxes).

\section{Limitations}

There are certain limitations of this study. The results of this study were obtained from a database with prospectively gathered data. However, this is a post hoc nonprespecified analysis and we cannot rule out the possibility that other potential confounding variables not included in the study might have affected the results. Furthermore, our findings are based on a small sample size and clinical events from a single site, which limits generalizability to the full spectrum of patients. With regard to the risk estimation model, clinical decision making solely based on our model would be pretentious. However, it may be used as an additional tool for both interdisciplinary heart team discussion and patient-orientated information.

\section{IMPLICATIONS FOR CLINICAL PRACTICE AND CONCLUSIONS}

With regard to intraprocedural complications, emergency conversion is associated with a significant mortality risk. However, CPB support for hemodynamic recompensation is a safe treatment option. Therefore, the heart team approach of performing TAVI in a hybrid operating room with a CPB standby represents a life-saving option in catheter-based valvular repair. Moreover, attention should also be drawn to specialized postoperative care, because even nonprocedure-specific complications (eg, pneumonia, abdominal complications, or transfusion) influence postoperative short- and long-term outcomes. In addition, further efforts to reduce postoperative AR may improve longterm outcome, and the risk-stratification model might facilitate reasonable preoperative decision making.

\section{Conflict of Interest Statement}

Authors have nothing to disclose with regard to commercial support.

\section{References}

1. Varadarajan P, Kapoor N, Bansal RC, Pai RG. Clinical profile and natural history of 453 nonsurgically managed patients with severe aortic stenosis. Ann Thorac Surg. 2006;82:2111-5.

2. Bonow RO, Carabello BA, Kanu C, de Leon AC Jr, Faxon DP, Freed MD, et al. ACC/AHA 2006 guidelines for the management of patients with valvular heart disease: a report of the American College of Cardiology/American Heart Association Task Force on Practice Guidelines (writing committee to revise the 1998 Guidelines for the Management of Patients With Valvular Heart Disease): developed in collaboration with the Society of Cardiovascular Anesthesiologists: endorsed by the Society for Cardiovascular Angiography and Interventions and the Society of Thoracic Surgeons. Circulation. 2006;114:e84-231.

3. Vahanian A, Alfieri OR, Al-Attar N, Antunes MJ, Bax J, Cormier B, et al. T. Transcatheter valve implantation for patients with aortic stenosis: a position statement from the European Association of Cardio-Thoracic Surgery (EACTS) and the European Society of Cardiology (ESC), in collaboration with the European Association of Percutaneous Cardiovascular Interventions (EAPCI). Eur J Cardiothorac Surg. 2008;34:1-8.

4. Kappetein AP, Head SJ, Genereux P, Piazza N, van Mieghem NM, Blackstone EH, et al. Updated standardized endpoint definitions for transcatheter aortic valve implantation: the Valve Academic Research Consortium-2 consensus document. Eur Heart J. 2012;33:2403-18.

5. Seiffert M, Schnabel R, Conradi L, Diemert P, Schirmer J, Koschyk D, et al. Predictors and outcomes after transcatheter aortic valve implantation using different approaches according to the Valve Academic Research Consortium definitions. Catheter Cardiovasc Interv. 2013;82:640-52.

6. Bleiziffer S, Ruge H, Mazzitelli D, Hutter A, Opitz A, Bauernschmitt R, et al. Survival after transapical and transfemoral aortic valve implantation: talking about two different patient populations. J Thorac Cardiovasc Surg. 2009;138:1073-80. 
7. Scherner M, Strauch JT, Haldenwang PL, Baer F, Wahlers T. Successful transapical aortic valve replacement in a patient with a previous mechanical mitral valve replacement. Ann Thorac Surg. 2009;88:1662-3.

8. Leon MB, Smith CR, Mack M, Miller DC, Moses JW, Svensson LG, et al. Transcatheter aortic-valve implantation for aortic stenosis in patients who cannot undergo surgery. $N$ Engl J Med. 2010;363:1597-607.

9. Kodali SK, Williams MR, Smith CR, Svensson LG, Webb JG, Makkar RR, et al. Two-year outcomes after transcatheter or surgical aortic-valve replacement. $N$ Engl J Med. 2012;366:1686-95.

10. Zahn R, Gerckens U, Grube E, Linke A, Sievert H, Eggebrecht H, et al. Transcatheter aortic valve implantation: first results from a multi-centre real-world registry. Eur Heart J. 2011;32:198-204.

11. Gurvitch R, Toggweiler S, Willson AB, Wijesinghe N, Cheung A, Wood DA, et al. Outcomes and complications of transcatheter aortic valve replacement using a balloon expandable valve according to the Valve Academic Research Consortium (VARC) guidelines. EuroIntervention. 2011;7:41-8.

12. Wenaweser P, Pilgrim T, Roth N, Kadner A, Stortecky S, Kalesan B, et al. Clinical outcome and predictors for adverse events after transcatheter aortic valve implantation with the use of different devices and access routes. Am Heart J. 2011;161:1114-24.

13. Seiffert M, Conradi L, Baldus S, Schirmer J, Blankenberg S, Reichenspurner H, et al. Severe intraprocedural complications after transcatheter aortic valve implantation: calling for a heart team approach. Eur J Cardiothorac Surg. 2013; 44:478-84; discussion 484.

14. Eggebrecht H, Schmermund A, Kahlert P, Erbel R, Voigtlander T, Mehta RH, et al. Emergent cardiac surgery during transcatheter aortic valve implantation (TAVI): a weighted meta-analysis of 9,251 patients from 46 studies. EuroIntervention. 2013;8:1072-80.

15. Smith CR, Leon MB, Mack MJ, Miller DC, Moses JW, Svensson LG, et al. Transcatheter versus surgical aortic-valve replacement in high-risk patients. $N$ Engl J Med. 2011;364:2187-98.
16. Gilard M, Eltchaninoff H, Iung B, Donzeau-Gouge P, Chevreul K, Fajadet J, et al Registry of transcatheter aortic-valve implantation in high-risk patients. $N$ Engl J Med. 2012;366:1705-15.

17. Tamburino C, Capodanno D, Ramondo A, Petronio AS, Ettori F, Santoro G, et al. Incidence and predictors of early and late mortality after transcatheter aortic valve implantation in 663 patients with severe aortic stenosis. Circulation. 2011;123:299-308

18. Thomas M, Schymik G, Walther T, Himbert D, Lefevre T, Treede H, et al. Thirtyday results of the SAPIEN aortic Bioprosthesis European Outcome (SOURCE) Registry: a European registry of transcatheter aortic valve implantation using the Edwards SAPIEN valve. Circulation. 2010;122:62-9.

19. Wendler O, Walther T, Schroefel H, Lange R, Treede H, Fusari M, et al. The SOURCE registry: what is the learning curve in trans-apical aortic valve implantation? Eur J Cardiothorac Surg. 2011;39:853-9; discussion 859-60.

20. Bagur R, Webb JG, Nietlispach F, Dumont E, De Larochelliere R, Doyle D, et al. Acute kidney injury following transcatheter aortic valve implantation: predictive factors, prognostic value, and comparison with surgical aortic valve replacement. Eur Heart J. 2010;31:865-74.

21. Elhmidi Y, Bleiziffer S, Piazza N. Incidence and predictors of acute kidney injury in patients undergoing transcatheter aortic valve implantation. Am Heart J. 2011 161:735-9.

22. Pasic M, Unbehaun A, Dreysse S, Drews T, Buz S, Kukucka M, et al. Transapical aortic valve implantation in 175 consecutive patients: excellent outcome in very high-risk patients. J Am Coll Cardiol. 2010;56:813-20.

23. Walther T, Simon P, Dewey T, Wimmer-Greinecker G, Falk V, Kasimir MT, et al. Transapical minimally invasive aortic valve implantation: multicenter experience. Circulation. 2007;116(11 Suppl):I240-5.

Key Words: TA-AVI, Tao-AVI, complications, predictors, outcome

\title{
EDITORIAL COMMENTARY
}

\section{Of mice and men and surgical transcatheter aortic valve insertion}

\author{
Kevin L. Greason, MD
}

From the Division of Cardiovascular Surgery, Mayo Clinic, Rochester, Minn.

Disclosures: Author has nothing to disclose with regard to commercial support.

Received for publication July 19, 2015; accepted for publication July 20, 2015; available ahead of print Aug 13, 2015.

Address for reprints: Kevin L. Greason, MD, Division of Cardiovascular Surgery, Mayo Clinic, 200 First St SW,

Rochester, MN 55905 (E-mail: greason.kevin@mayo.edu).

J Thorac Cardiovasc Surg 2015;150:849-50

$0022-5223 / \$ 36.00$

Copyright (C) 2015 by The American Association for Thoracic Surgery

http://dx.doi.org/10.1016/j.jtcvs.2015.07.060

The best-laid schemes of Mice and Men/go oft awry — Robert Burns (1759-1796) ${ }^{1}$

Readers of the Journal will find interest in article by Scherner and colleagues ${ }^{2}$ about transapical and transaortic transcatheter aortic valve insertion. The authors have christened the procedures "surgical transcatheter aortic valve insertion." I like the term and think it is an appropriate moniker. The objectives of the study were as follows: (1) to identify periprocedural complications and their impact on 30-day and 1-year survivals and (2) to develop a risk stratification model of

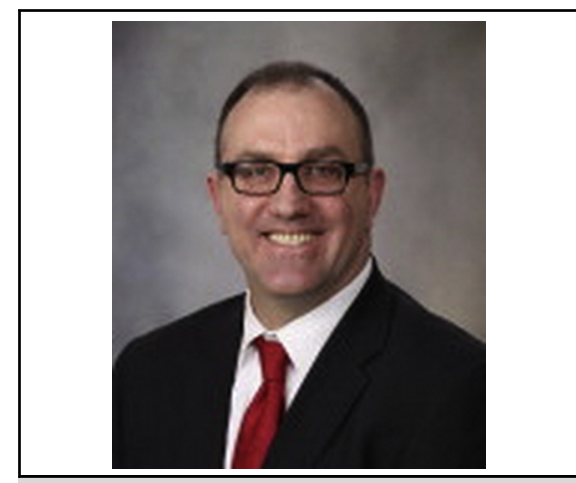

Kevin L. Greason, MD

Central Message

Early mortality is procedure related; late mortality is baseline patient characteristic related.

See Article page 841 . 